\title{
Self-similar clustering of inertial particles and zero-acceleration points in fully developed two- dimensional turbulence
}

$\operatorname{AUTHOR}(S)$ :

Goto, Susumu; Vassilicos, J.C.

\section{CITATION:}

Goto, Susumu ...[et al]. Self-similar clustering of inertial particles and zero-acceleration points in fully developed two-dimensional turbulence. PHYSICS OF FLUIDS 2006, 18(11): 115103.

\section{ISSUE DATE:}

2006-11

URL:

http://hdl.handle.net/2433/39788

\section{RIGHT:}

Copyright 2006 American Institute of Physics. This article may be downloaded for personal use only. Any other use requires prior permission of the author and the American Institute of Physics. 


\title{
Self-similar clustering of inertial particles and zero-acceleration points in fully developed two-dimensional turbulence
}

\author{
Susumu Goto ${ }^{\text {a) }}$ \\ Department of Mechanical Engineering and Science, Kyoto University, Yoshida-Honmachi, Sakyo, \\ Kyoto 606-8501, Japan \\ J. C. Vassilicos ${ }^{\text {b) }}$ \\ Department of Aeronautics, Imperial College, London SW7 2AZ, United Kingdom \\ and Institute for Mathematical Sciences, Imperial College, London SW7 2PE, United Kingdom
}

(Received 31 May 2006; accepted 26 September 2006; published online 20 November 2006)

\begin{abstract}
Clustering of inertial particles in fully developed two-dimensional inverse cascading turbulence occurs for all particle relaxation times ranging from an order of magnitude under the smallest eddy turnover time to an order of magnitude above the largest eddy turnover time. Particle voids and clusters are statistically self-similar over a finite range of scales within the inertial range and are explained in terms of coarse-grained vorticity and resonant eddies (for voids) and in terms of zero-acceleration points (for clusters). The clustering of inertial particles reflects the clustering of zero-acceleration points. Essential to both explanations is the sweeping of small eddies by large ones. An important implication is that particle clustering can be explicitly described just in terms of the fluid acceleration field without the need for Lagrangian particle integrations. () 2006 American
\end{abstract} Institute of Physics. [DOI: 10.1063/1.2364263]

\section{INTRODUCTION}

The preferential concentration (clustering) of small heavy particles in turbulence is a well-known phenomenon, with important implications in various environmental (see, e.g., Ref. 1) and industrial (see, e.g., Ref. 2) flows, and it has been explained ${ }^{3-5}$ by the centrifugal effect of coherent intense smallest-scale eddies that can be found even in homogeneous isotropic turbulence. These studies have also claimed that clustering is most significant when the relaxation time $\tau_{p}$ [see (1) below for its definition] of inertial particles is comparable to the Kolmogorov time, i.e., the typical time scale of swirling motion of the smallest eddies.

However, it has been pointed out by Boffetta et al. ${ }^{6}$ that a multiscale structure of clustering can be observed in fully developed isotropic homogeneous two-dimensional turbulence in the inverse energy cascade regime. In turbulence with the Kolmogorov $-5 / 3$ power-law spectrum, most of the enstrophy is at the smallest scales. It is therefore the smallest eddies that are observed predominantly if one focuses on vorticity. At the same time, however, in such fully developed turbulence, multiscale eddies do exist simultaneously, and each one of them might contribute to the clustering of the inertial particles if $\tau_{p}$ is comparable to their swirling time scales.

One purpose of the present article is to demonstrate this picture of multiscale clustering due to multiscale coherent eddies with the help of direct numerical simulations (DNS) of two-dimensional turbulence, and to develop a physical model to explain the self-similarity of inertial particle clustering based on this picture. Note that our approach and ar-

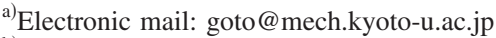

${ }^{b)}$ Electronic mail: j.c.vassilicos@imperial.ac.uk
}

gument are not inconsistent with previous numerical ${ }^{3,4}$ and experimental $^{5}$ works on moderate Reynolds number turbulent flows, since the largest and the smallest scales are not well separated in such not-fully-developed turbulence, implying that the multiscale nature of the velocity field, and therefore of inertial particle clustering, is not significant.

Another purpose of the present article is to bridge the gap between explanations of particle clustering based on vorticity and our recent demonstration in Ref. 7 that particle clustering mirrors the clustering of zero-acceleration points. This gap is bridged here because our approach based on the multiscale structure of coherent eddies is an extension of the conventional picture based on the smallest-scale eddies only. An incomplete explanation of the coincidence of particle and zero-acceleration point clusters was given in Ref. 7, which we develop here and complete. Essential to this explanation is the "stickiness" of zero-acceleration points and their sweeping by the local fluid velocity.

\section{INERTIAL PARTICLES IN FULLY DEVELOPED TURBULENCE}

\section{A. Equation of motion of inertial particles}

We consider particles that are small heavy rigid spheres all of the same size. Their mass density $\rho_{p}$ is assumed to be much heavier than the fluid density, and their radius $a$ is sufficiently small for the Reynolds number based on the particle radius being much smaller than unity and the Stokes approximation holding for the local flow around each particle. The radius is also assumed to be smaller than the smallest length scale of the turbulence. Under these assumptions, and ignoring gravity, the equation of motion for a particle is ${ }^{8}$ 
TABLE I. Statistics of turbulent velocity field. $N^{2}$, the number of grid points; $\mathcal{L}$, integral scale; $\eta$, forcing scale; $u^{\prime}$, rms velocity; $\mathcal{T} \equiv \mathcal{L} / u^{\prime}$, eddy turnover time; $\epsilon$, mean energy flux in the inertial range; $\tau_{\eta}$, Kolmogorov time determined by the rms vorticity as $\tau_{\eta} \equiv \omega^{\prime-1}$.

\begin{tabular}{lrrrrrrrr}
\hline \hline & $N^{2}$ & $\mathcal{L}$ & $\mathcal{L} / \eta$ & $u^{\prime}$ & $\mathcal{T}$ & $\epsilon$ & $\tau_{\eta}$ & $\mathcal{T} / \tau_{\eta}$ \\
\hline run A & $512^{2}$ & 0.38 & 6.3 & 3.2 & 0.12 & 3.0 & 0.012 & 10 \\
run D & $4096^{2}$ & 0.23 & 30 & 1.3 & 0.17 & 0.20 & 0.0068 & 25 \\
\hline \hline
\end{tabular}

$$
\frac{\mathrm{d}}{\mathrm{d} t} \boldsymbol{v}_{p}(t)=\frac{1}{\tau_{p}}\left[\boldsymbol{u}\left(\boldsymbol{x}_{p}(t), t\right)-\boldsymbol{v}_{p}(t)\right] .
$$

Here, $\boldsymbol{v}_{p}(t)$ and $\boldsymbol{u}(\boldsymbol{x}, t)$ are the velocities of an inertial particle at position $\boldsymbol{x}_{p}(t)$ and of the fluid at position $\boldsymbol{x}$ and time $t$, respectively. This equation implies that the particle velocity relaxes to the fluid velocity within a time scale $\tau_{p}$. According to the Stokes approximation, $\tau_{p}=2 \rho_{p} a^{2} /(9 \mu)$, where $\mu$ is the fluid viscosity. We further assume that particles do not affect fluid motion, and that the number density of particles is small enough for interactions between particles to be neglected. All these neglected effects might be important in real flows, but we leave them for future study because even when we neglect them, the system described by (1) is sufficiently complex and worth studying as a foundation for the future.

\section{B. Self-similarity in fully developed turbulence}

Being interested in the statistics of inertial particle clustering in fully developed turbulence, we consider a velocity field $\boldsymbol{u}(\boldsymbol{x}, t)$ in (1) that is two-dimensional and fully turbulent because we can simulate numerically two-dimensional turbulence at much higher Reynolds numbers than threedimensional turbulence. Here, we numerically integrate turbulence governed by the Navier-Stokes equation with an external small-scale energy source and a large-scale energy sink. Periodic boundary conditions for the velocity field are imposed in two orthogonal directions. In this system, the energy cascades from small to large scales, ${ }^{9-11}$ and the energy flux settles at a negative constant, $-\epsilon$, in the inertial range between the small forcing scale $\eta$ (hereafter, we call $\eta$ the Kolmogorov length) and the integral length $\mathcal{L}$ (of the second-order longitudinal velocity correlation function) where the energy spectrum takes the power-law form

$$
E(k) \sim \epsilon^{2 / 3} k^{-5 / 3} .
$$

Details of this DNS are given in Ref. 12. By using up to $4096^{2}$ grid points, we simulate such turbulence over almost two decades in wave-number space (see Figs. 1 and 2 of Ref. 12). The statistics of the simulated turbulent flows are listed in Table I. In this article, we use two cases; one is a small $\mathcal{L} / \eta$ case (Run A) and the other is a large $\mathcal{L} / \eta$ case (Run D). Note that in this two-dimensional turbulence, the value $\mathcal{L} / \eta$ serves as an index of how developed the turbulence is, and we call $\mathcal{L} / \eta$ the Reynolds number. Of course note that, strictly speaking, this is not a real Reynolds number as $\eta$ is not a Kolmogorov length and that the real Reynolds number in three-dimensional isotropic turbulence is proportional to $(\mathcal{L} / \eta)^{4 / 3}$, where $\eta$ is the Kolmogorov length. Our use of the term "Reynolds number" is by analogy.

One of the most important characteristics of fully developed turbulence is that motions of a wide range of length scales are excited simultaneously. More precisely, in the case of an energy spectrum with a power-law form such as (2), eddies of many length scales between $\eta$ and $\mathcal{L}$ coexist, and eddies of a given length scale are advected by eddies larger than them. Note that larger-scale eddies are more energetic, though smaller-scale eddies are more conspicuous because the enstrophy spectrum $k^{2} E(k)$ peaks in the high wavenumber range while $E(k)$ peaks in the low wave-number region. This multiscale nature of turbulent eddies is captured well by the coarse-grained vorticity field $\omega_{c}\left(\boldsymbol{x}, t \mid k_{c}\right)$ obtained by low-pass filtering Fourier components of vorticity with a sharp cutoff at wave number $k_{c}$. It is observed in Fig. 1 (a movie is also available online) that these eddies are spinning with the time scale $\left|\omega_{c}\right|^{-1}$, and are swept by eddies larger than them. This multiscale nature of spinning and swept coherent eddies plays a crucial role in the clustering of inertial particles.

\section{Scale-dependent Stokes number}

For scaling purposes, the relaxation time $\tau_{p}$ in (1) should be normalized by a time scale of the turbulence. This normalized relaxation time is called the Stokes number, often defined as
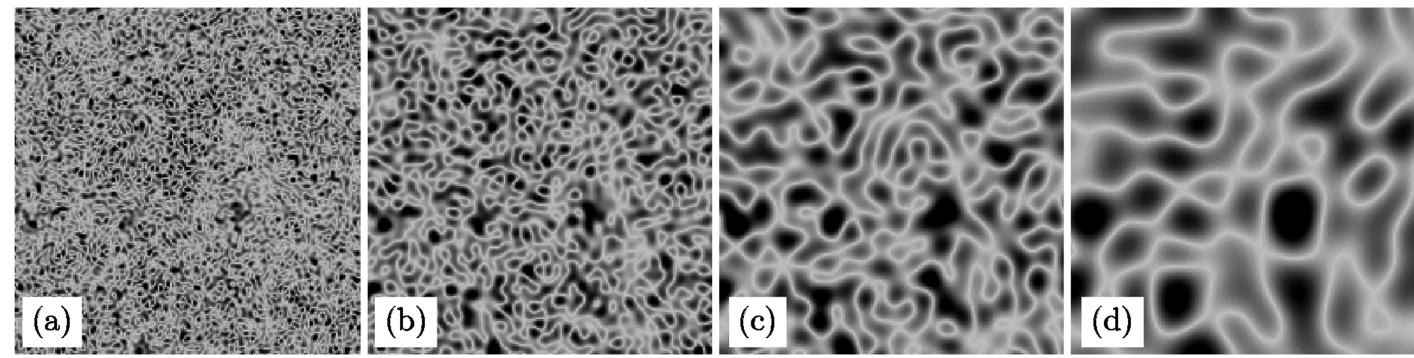

FIG. 1. Coarse-grained vorticity fields (the magnitudes of which are plotted in shades of gray) by low-pass filtering Fourier components of vorticity with a sharp cutoff at wave number $k_{c}$. (a) $k_{c}=k_{\eta} / 4$, (b) $k_{\eta} / 8$, (c) $k_{\eta} / 16$, and (d) $k_{\eta} / 32$. Here, $k_{\eta} \equiv 2 \pi / \eta$. The side length of the plots is about $7 \mathcal{L} \approx 200 \eta$. Run D (enhanced online). 


$$
S_{\eta} \equiv \frac{\tau_{p}}{\tau_{\eta}}
$$

which is the relaxation time normalized by the Kolmogorov time $\tau_{\eta}$. [The average stretching rate of infinitesimal material line elements, $\gamma$ (see Ref. 17 for details on how $\gamma$ is calculated) in this two-dimensional turbulence is $0.22 \tau_{\eta}^{-1}$. Hence, the Stokes number $S_{b} \equiv \tau_{p} \gamma$ used by Boffetta et al. ${ }^{6}$ is $S_{b}$ $=0.22 S_{\eta}$. In this article's arguments, it is useful to define generalized scale-dependent Stokes numbers,

$$
S(\ell) \equiv \frac{\tau_{p}}{T(\ell)}
$$

where

$$
T(\ell) \sim \frac{\ell}{\sqrt{E(1 / \ell) / \ell}} \sim \epsilon^{-1 / 3} \ell^{2 / 3}
$$

is the turnover time of eddies of size $\ell$. Here, we have assumed (2). The constant of proportionality in (5) is determined so that $T(\eta)=\tau_{\eta}$, i.e., $T(\ell)=0.11 \epsilon^{-1 / 3} \ell^{2 / 3}$ irrespective of the Reynolds number $\mathcal{L} / \eta$.

This scale-dependent Stokes number $S(\ell)$ is useful because of the multiscale nature of turbulent eddies as the following argument shows. If $S(\ell) \ll 1$, then inertial particles promptly follow the motion induced by eddies of size $\ell$, and therefore no clustering may be brought about by such eddies. On the other hand, if $S(\ell) \gg 1$, then inertial particles completely ignore the fluid motion induced by eddies of size $\ell$, and therefore no clustering of size $\ell$ takes place. Hence, only when $S(\ell)$ is not too far from 1 do eddies of size $\ell$ play a role in the clustering of inertial particles. Since eddies of many different sizes coexist in fully developed turbulence, we expect eddies of many length scales such that $S(\ell)$ $=O(1)$ [a more precise condition is given in (8) below] to contribute simultaneously to the clustering of inertial particles. Therefore, the resultant clustering must have a selfsimilar feature if the velocity field also has one. As will be shown below, such self-similar clustering is indeed observed in the present numerical simulation.

\section{SELF-SIMILAR CLUSTERING OF INERTIAL PARTICLES}

\section{A. Stokes number and Reynolds number dependence of particle clustering}

We plot in Fig. 2 the spatial distribution of $2^{18} \approx 10^{5}$ inertial particles originally uniformly distributed and released in turbulence of Reynolds number $\mathcal{L} / \eta=6.3$ (Run A). The snapshots in Fig. 2 are taken at time $t=8.3 \mathcal{T}$ for nine different Stokes numbers ranging from (a) $S_{\eta}=0.1$ to (i) 25.6. The size of the box is $10 \mathcal{L}(\approx 63 \eta)$. When $S_{\eta}$ is as small as 0.1 , i.e., Fig. 2(a), all void regions (holes) characterizing the clustering are as small as $O(\eta)$. As $S_{\eta}$ becomes larger, larger holes appear. The size of the largest holes seems to saturate around Fig. 2(e) $S_{\eta}=1.6$ or Fig. 2(f) 3.2. Observe that, at this Stokes number, not only the conspicuous large holes but also small holes exist, and that the size of the largest holes is $O(\mathcal{L})$. This observation is consistent with the results of
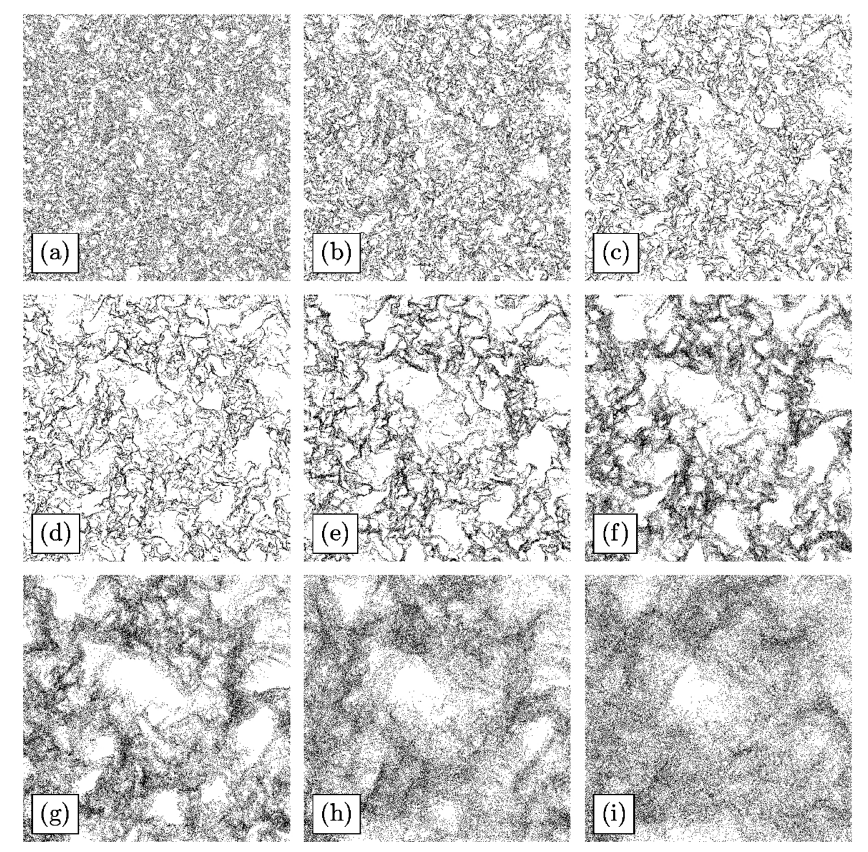

FIG. 2. Spatial distribution of inertial particles. Run A. $t=8.3 \mathcal{T}$. The side length of the plots is $10 \mathcal{L}(\approx 63 \eta)$. (a) $S_{\eta}=0.1$, (b) 0.2 , (c) 0.4 , (d) 0.8 , (e) 1.6 , (f) 3.2, (g) 6.4 , (h) 12.8 , and (i) 25.6 .

Boffetta et al. $;^{6}$ that is, the probability density function (PDF) of hole areas takes a power-law form, and the cutoff of the power law depends on the Stokes number. This powerlaw form of the PDF is also confirmed in this field (see Fig. 7). As $S_{\eta}$ increases further, holes of small scales become fainter and eventually disappear. When $S_{\eta}=25.6$ [i.e., $S(\mathcal{L})$ $=2.56]$, even the largest holes become faint.
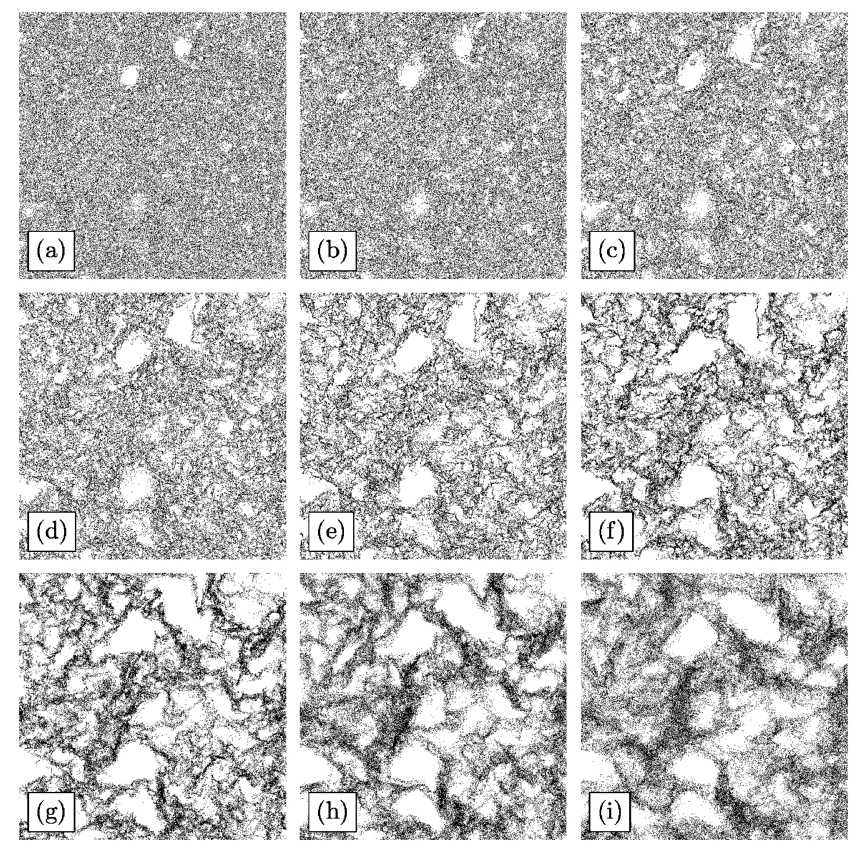

FIG. 3. Spatial distribution of inertial particles. Run D. $t=3.8 \mathcal{T}$. The side length of the plots is $10 \mathcal{L}(\approx 300 \eta)$. (a) $S_{\eta}=0.1$, (b) 0.2 , (c) 0.4 , (d) 0.8 , (e) 1.6, (f) 3.2, (g) 6.4 , (h) 12.8, and (i) 25.6. 


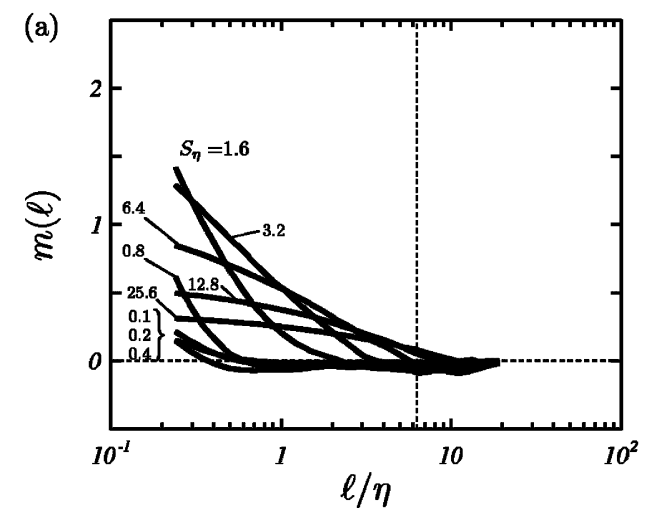

FIG. 4. Pair correlation function $m(\ell)$ for nine different Stokes numbers $(0.1$, $0.2,0.4, \ldots, 25.6)$. Each curve is the result of an average over 100 snapshots. Vertical lines indicate integral length $\mathcal{L}$. (a) Run A. (b) Run D.
Particle distributions for a larger value of $\mathcal{L} / \eta=30$ (Run D) are plotted in Fig. 3. The number of particles is $2^{20}$ $\approx 10^{6}$, and the snapshots shown are at $t=3.8 \mathcal{T}$. Similar features to the lower $\mathcal{L} / \eta$ case are observed; (I) when $S_{\eta}$ is small, only small holes are observed, (II) as $S_{\eta}$ increases, larger holes appear, (III) the size of the largest holes saturates at $O(\mathcal{L})$ when $S_{\eta}$ is around Fig. 3(f) 3.2 or Fig. 3(g) 6.4; (IV) as $S_{\eta}$ increases further, smaller holes start to lose their definition and eventually disappear. Compared to the lower $\mathcal{L} / \eta$ case, the larger holes can survive even at large $S_{\eta}$. Note that $S_{\eta}=25.6$ corresponds to $S(\mathcal{L})=1.0$ in Run D.

The above observations can be quantified by the pair correlation function $m(\ell)$. Following Ref. 13 and Sec. 116 of Ref. 14, we numerically estimate the pair correlation function $m(\ell)$ of the spatial distribution of inertial particles using the formula

$$
\frac{1}{\ell} \int_{0}^{\ell} m\left(\ell^{\prime}\right) \mathrm{d} \ell^{\prime}=\frac{\left\langle\left(N-\langle N\rangle_{\ell}\right)^{2}\right\rangle_{\ell}}{\langle N\rangle_{\ell}^{2}}-\frac{1}{\langle N\rangle_{\ell}},
$$

where the integral on the left-hand side is taken over a box of size $\ell^{2}$, and brackets \langle\rangle$_{\ell}$ denote the average over many boxes of size $\ell^{2}$, e.g.,

$$
\langle N\rangle_{\ell}=\frac{1}{I} \sum_{i=1}^{I} N^{(i)},
$$

where $N^{(i)}$ is the number of particles that are in the $i$ th box of size $\ell^{2}$. Note that the right-hand side of (6) vanishes for Poisson spatial distributions. We plot $m(\ell)$ in Fig. 4, which is estimated by differentiating the numerical value of the righthand side of (6). The qualitative observations we made in Figs. 2 and 3 are also captured by $m(\ell)$. Furthermore, $m(\ell)$ can highlight differences that are not clear in the visualizations; for example, the difference between (d) $S_{\eta}=0.8$, (e) 1.6, and (f) 3.2 in Fig. 3 is clearly reflected in the gradual increase of $m(\ell)$ in Fig. 4(b). In both cases of $\mathcal{L} / \eta$, when $S_{\eta}$ is smaller than $O(1), m(\ell)$ vanishes for $\ell>\eta$. This agrees with the absence of clustering characterized by holes larger than $\eta$. Only the smallest scale holes of $O(\eta)$ exist when $S_{\eta} \lesssim 1$. As $S_{\eta}$ increases, the pair correlation takes nonzero values in the inertial range and rises until $S_{\eta}$ reaches 1.6 in Run A and around 3.2 in Run D. As $S_{\eta}$ increases further, $m(\ell)$ gradually returns to smaller values. This decrease of $m(\ell)$, for larger $S_{\eta}$, begins from the smaller $\ell$ range.

\section{B. Physical explanation of the self-similar clustering}

The hypothesis drawn from the observations in Figs. 2-4 is that inertial particle clustering is governed by eddies of size $\ell$, which satisfy a resonance condition with the relaxation time scale $\tau_{p}$ of particles. This picture in terms of the resonance between particles and eddies can be summarized as follows. Particles promptly follow the slow motion induced by large eddies with swirling time $T(\ell) \gg \tau_{p}$ [i.e., $S(\ell) \ll 1$ ], but also completely ignore the fast motion induced by small eddies with $T(\ell) \ll \tau_{p}$ [i.e., $S(\ell) \gg 1$ ]. Thus, particles are affected by eddies of size $\ell$ that satisfy

$$
\alpha<S(\ell)<\beta .
$$

Here, $\alpha$ and $\beta$ are constants and $S(\ell)$ is the scale-dependent Stokes number (4). Therefore, the smallest and the largest sizes of the eddies that satisfy the resonance condition (8) are

$$
\ell_{\min }=\beta^{-3 / 2} S_{\eta}^{3 / 2} \eta
$$

and

$$
\ell_{\max }=\alpha^{-3 / 2} S_{\eta}^{3 / 2} \eta
$$

respectively. All the eddies with length scales ranging between $\ell_{\min }$ and $\ell_{\max }$ impact on the clustering of particles.

It is worth mentioning that the above hypothesis in terms of multiscale eddies is an extension of the conventional picture based on the smallest-scale eddies' centrifugal effects on particles. However, our picture is significantly different. Indeed, we have shown ${ }^{7}$ that when $S_{\eta}$ is sufficiently larger than 1, particle distributions cannot be explained in terms of bare vorticity or strain rate only. Velocity gradients reflect the smallest scales only, but it is eddies of scales throughout the inertial range that impact on particle clustering when $S_{\eta} \gg 1$.

It is also important to mention that for the centrifugal effect of eddies to have an impact on inertial particles, it is necessary that particles stay within these eddies for long enough times. This is indeed the case because of the turbulence sweeping whereby eddies are swept by larger eddies that also sweep the particles with them. Hence, in a Lagrangian frame moving with the sweeping velocity induced by the larger eddies, particles stay within resonant eddies long enough to be centrifuged out of them.

The pair correlation function plotted in Fig. 4 allows us to estimate the two constants $\alpha$ and $\beta$ in (8). Irrespective of 

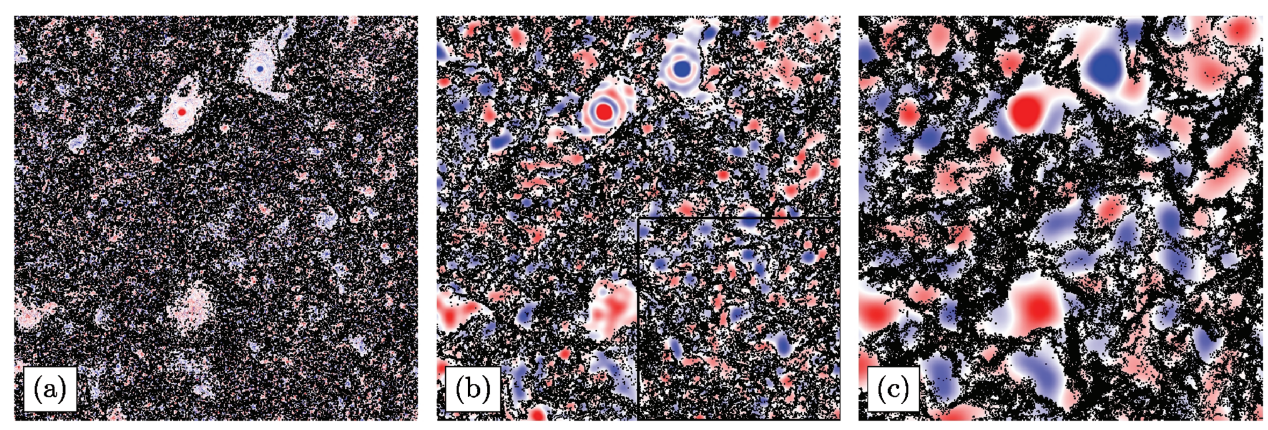

Phys. Fluids 18, 115103 (2006) FIG. 5. (Color) (a) Spatial distribution
of inertial particles $\left(S_{\eta}=0.4\right)$ and the
magnitude of coarse-grained vorticity
field by sharp low-pass filtering at $k_{c}$
$=0.83 k_{\eta}$. The side length of the plots
is $10 \mathcal{L}$. Positive/negative vorticity re-
gions are colored by red/blue. Run D.
$\begin{array}{ll}\text { (b) } S_{\eta}=1.6, k_{c}=0.093 k_{\eta} & \text { (c) } S_{\eta}=6.4 \text {, } \\ k_{c}=0.037 k_{\eta} . & \end{array}$ the Reynolds number $\mathcal{L} / \eta$, when $S_{\eta}$ is smaller than $0.4, m(\ell)$ is independent of $S_{\eta}$. This implies that when $\tau_{p} \subseteq 0.4 \tau_{\eta}$, there is only negligible action by most eddies. On the other hand, when $\tau_{p}=0.8 \tau_{\eta}$, the smallest eddies have a clear impact on the preferential concentration, and $m(\ell)$ (for $\ell \leqq \eta$ ) grows clearly above its values at $S_{\eta} \leq 0.4$. Therefore, we may roughly estimate $\alpha$ as 0.6 , which is between 0.4 and 0.8 . Concerning $\beta$, looking at Fig. 4 again we notice that the correlation function $m(\ell)$ at $\ell=\eta$ takes its maximum value when $S_{\eta}=6.4$ in both cases of $\mathcal{L} / \eta$. This means that eddies of size $\eta$ cease to contribute to the particle clustering when $\tau_{p}$ is larger than $6.4 \tau_{\eta}$. Therefore, the upper bound of (8) is given as $\beta \approx 6$. The two constants in (8) are thus determined roughly as

$$
\alpha \approx 0.6, \quad \beta \approx 6
$$

for the two-dimensional turbulence.

In order to verify the above argument qualitatively, we plot in Fig. 5 inertial particles together with the coarsegrained vorticity field, which is obtained by sharp low-pass filtering of Fourier components of vorticity at the cutoff wave number $k_{c}=\widetilde{k}_{c}\left(S_{\eta}\right)$. Here, $\widetilde{k}_{c}$ is chosen so that the conditional average $\left\langle\omega_{c}\left(\boldsymbol{x}, t \mid k_{c}\right)\right\rangle_{p}$ of the magnitude of coarsegrained vorticity at positions of inertial particles takes a minimum value when $k_{c}=\tilde{k}_{c}\left(S_{\eta}\right)$. It is numerically shown that $\tilde{k}_{c} \approx 0.5 S_{\eta}^{-3 / 2} \eta^{-1}$, consistent with the low-pass filtering argument of Ref. 15 as well as (10). Since it is the largest eddies of size $\ell_{\max }$ that predominantly contribute to $\left\langle\omega_{c}\right\rangle_{p}$, we expect $\tilde{k}_{c}^{-1} \approx \ell_{\max } /(2 \pi)$.

Note that, in Fig. 5, many smaller holes of inertial particles are observed between the conspicuous largest holes, and these are not explained by the centrifugal effect of the eddies of size $\ell_{\max }$ only but also of all the smaller eddies of size larger than $\ell_{\min }$. This is demonstrated in Fig. 6, where we magnify Fig. 5(b) two and four times $\left(k_{c}\right.$ is also enlarged two and four times). In these figures, we observe the selfsimilarity of inertial particle clustering; Fig. 6(b) is the magnification of clusters of inertial particles in Fig. 6(a), and Fig. 6(c) is the magnification of clusters of inertial particles in Fig. 6(b). Smaller holes exist between larger holes. We also observe that particles avoid high-intensity regions of coarsegrained vorticity at each length scale. This implies that the multilength nature of particle holes is likely to be explained in terms of self-similar coherent eddies; i.e., larger/smaller holes are due to larger/smaller eddies.

Thus, the self-similarity of particle clustering is due to the self-similarity of coherent eddies, which satisfy the resonance condition (8), as verified roughly by numerical visualization. In the following two subsections, we develop a quantitative argument based on this self-similarity.

\section{Quantification of the self-similar clustering}

A method for quantifying the multiscale nature of inertial particle clustering is by way of measuring its fractal dimensions. However, after trying to calculate the boxcounting dimension of the particles' spatial distribution, we found that the inertial range of our present DNS, although relatively wide, is nevertheless not wide enough for a good and unambiguous estimation of the box-counting dimension. For this reason, we follow Ref. 6 in defining voids as connected regions of empty boxes from a grid of boxes covering all space (the size of the box being defined by the resolution of the simulation) and then calculating the PDF, $P(A)$, of void areas $A$. We plot this PDF in Fig. 7 for two different Reynolds numbers. It is clearly seen in this figure that as $S_{\eta}$ increases, larger holes appear, and that $P(A)$ takes a clear power-law form when $S_{\eta}=O(1) \sim O(10)$. The scaling exponent lies between -1.6 and $-2.0{ }^{6}$ Figure 7 suggests that this
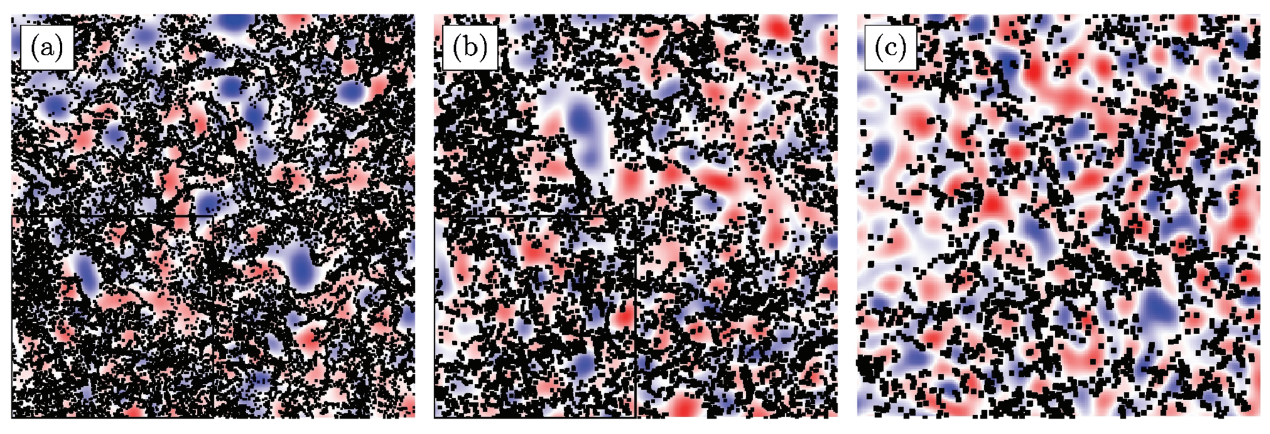

FIG. 6. (Color) (a) Same as Fig. 5(b) but only the lower right quarter is shown. (b) The magnification of the lower left quarter of (a). $k_{c}=0.19 k_{\eta}\left(=2 \widetilde{k}_{c}\right)$. (c) The magnification of the lower left quarter of (b). $k_{c}=0.37 k_{\eta}\left(=4 \tilde{k}_{c}\right)$. 

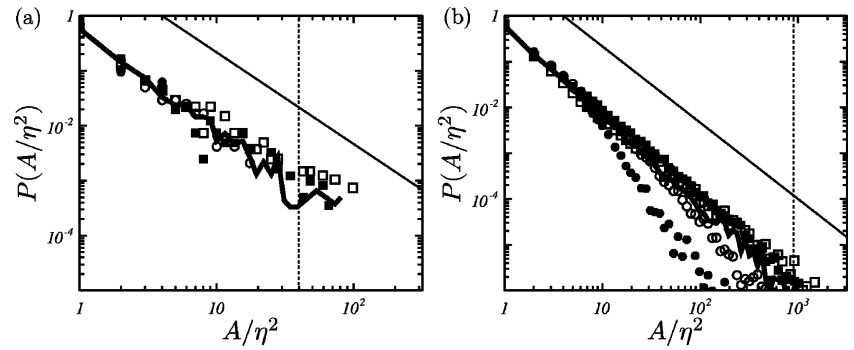

FIG. 7. PDF of hole areas $A$ of inertial particles. $\bullet, S_{\eta}=0.1 ; \bigcirc, 0.4 ; \mathbf{\square}, 1.6$; $\square$, 6.4. Thick solid curve is the PDF of hole areas of zero-acceleration points. Solid straight lines indicate the scaling $A^{-5 / 3}$ predicted by the model developed in Sec. III D, and vertical dotted lines indicate $A=\mathcal{L}^{2}$. (a) Run A. (b) Run D.

exponent is independent of $\mathcal{L} / \eta$, and we expect the range over which it is well-defined to be always the same and limited by $\ell_{\min }$ and $\ell_{\max }$ as long as $\mathcal{L} / \eta$ is much larger than $\ell_{\max } / \ell_{\min }$. This scaling behavior of $P(A)$ implies that the hole sizes of inertial particle clusters are never described by a single scale (e.g., the Kolmogorov length) structure, and that the inertial particle clustering is self-similar, reflecting the self-similarity of background turbulence within the resonance window of scales between $\ell_{\min }$ and $\ell_{\max }$. This is meaningful because $\beta / \alpha$ is $O(10)$, and therefore $\ell_{\max } / \ell_{\min }$ is not small.

The above result strengthens the arguments in our previous subsections. Incidentally, it has been shown in Ref. 7 that the pair correlation function $m(\ell)$ takes a power-law form (though its exponent depends on $S_{\eta}$ ), which also implies selfsimilarity of particle clustering.

\section{Model to explain the self-similarity of void areas}

In this subsection, we present a simple model to explain the power-law form of $P(A)$. This model is based on the fact that turbulence with the energy spectrum (2) has a selfsimilar structure of coherent eddies (Fig. 1). Inertial particles are swept out of these eddies, and $P(A)$ obeys a power law because hole sizes are self-similarly distributed in a finite range determined by $\ell_{\min }$ and $\ell_{\max }$ and particles are swept out of such sized eddies (Fig. 6). More precisely, the largest holes are due to the largest-scale eddies. Then, in regions where the largest-scale eddies are absent, the second-largest eddies eject inertial particles. Hence, the second-largest holes are due to these eddies. Then, in the rest of space, where the largest and the second-largest eddies are absent, next-scale eddies eject particles, and so on.

Based on this picture, a simple model of inertial particle clustering can be constructed. First, we assume that the turbulent velocity field consists of eddies at discrete scales,

$$
\ell_{m} \equiv \mathcal{L} \xi^{-m}
$$

with

$$
m=0,1,2, \ldots, M .
$$

Here, $\ell_{M} \approx \eta$, and therefore $M$ depends on the Reynolds number, and $\xi(>1)$ is a constant. Second, eddies at each scale, $\ell_{m}$, are assumed to occupy a ratio $R$ of space. Here, we assume, from self-similarity, that $R$ is independent of $m$. According to our clustering picture and following from the first assumption, holes have discrete areas $\ell_{m}^{2}(m=0,1, \ldots, M)$. Under these two assumptions, the PDF of the hole area can be estimated as follows.

Let us start with the largest scale in the turbulence. By definition of $R$, the total area $A_{0}^{\text {total }}$ of holes of area $\ell_{0}^{2}$ is $R L_{0}^{2}$. Here, $L_{0}$ is the system size. Then, since the rest of the space is $(1-R) L_{0}^{2}$, the total area $A_{1}^{\text {total }}$ of holes of area $\ell_{1}^{2}$ is $R(1$ $-R) L_{0}^{2}$. Inductively, the total area of holes of area $\ell_{m}^{2}$ is

$$
A_{m}^{\text {total }}=R(1-R)^{m} L_{0}^{2} .
$$

Then, the number $N_{m}$ of holes of size $A_{m}=\ell_{m}^{2}=\mathcal{L}^{2} \xi^{-2 m}$ is

$$
N_{m}=\frac{A_{m}^{\text {total }}}{A_{m}}=\frac{L_{0}^{2} R(1-R)^{m}}{A_{m}} \sim A_{m}^{-1-(1 / 2) \log _{\xi}(1-R)} .
$$

Consequently, the PDF of $A$ takes the form

$$
P(A) \sim A^{-2-(1 / 2) \log _{\xi}(1-R) .}
$$

Here, the extra $A^{-1}$ factor stems from the definition of $A_{m}$; note that $A_{m}$ are equidistantly located on the logarithmic $A$ axis.

In the above argument, we have introduced two artificial parameters, $\xi$ and $R$. However, they are not independent. As we are considering turbulence with the Kolmogorov spectrum (2), the energy in the eddies between scales $\ell_{m}$ and $\ell_{m+1}$ is

$$
\int_{\ell_{m}^{-1}}^{\ell_{m+1}^{-1}} E(k) \mathrm{d} k \sim \epsilon^{2 / 3} \ell_{m}^{2 / 3}\left[1-\xi^{-2 / 3}\right] .
$$

Hence, in the discrete model introduced above, the $m$ th scale eddies occupy a portion of space proportional to the factor $1-\xi^{-2 / 3}$. Since $R$ must tend to 1 in the limit where $\xi$ tends to infinity, we may conclude

$$
R=1-\xi^{2 / 3}
$$

for turbulence with the Kolmogorov spectrum. Then, substituting (18) into (16), we have

$$
P(A) \sim A^{-5 / 3} \text {. }
$$

The scaling observed in Fig. 7 supports this consequence of our simple model.

\section{COINCIDENCE WITH ZERO-ACCELERATION CLUSTERING}

The remaining problem is where inertial particles, which are swept out of multiscale coherent eddies, accumulate. Because eddies of different sizes are effectively superimposed on each other in a complex way, it is very difficult to define the regions where particles cluster in term of multiscale eddies or even in terms of multiscale strain fields. Instead, the particle clusters can be easily described in terms of zeroacceleration points. As shown in our previous paper, ${ }^{7}$ the clustering of inertial particles reflects that of zeroacceleration points. In this section, we develop the argument explaining this coincidence. 


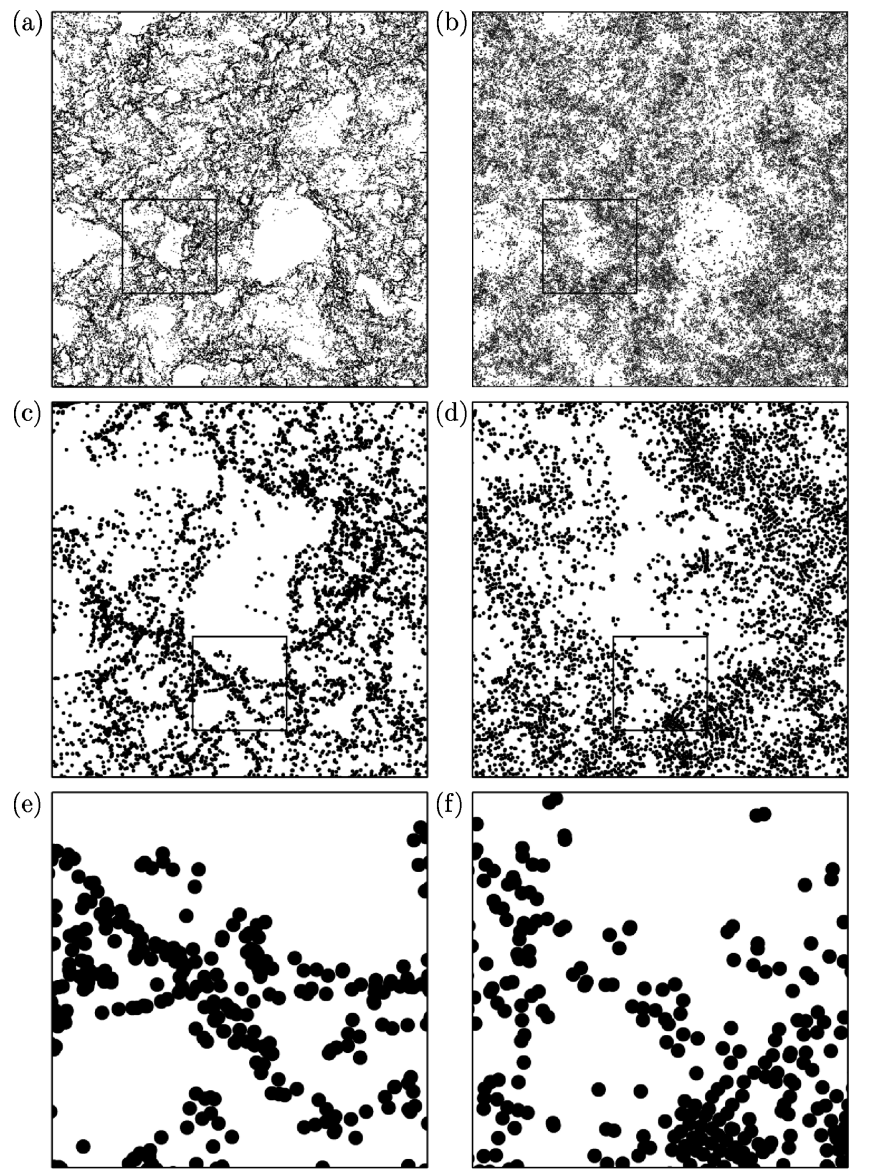

FIG. 8. [(a), (c), and (e)] Spatial distribution of inertial particles. $S_{\eta}=1.9$. $[(\mathrm{b}),(\mathrm{d})$, and (f)] Spatial distribution of zero-acceleration points. [(a) and (b)] The side length of the plots is about $7 \mathcal{L} \approx 200 \eta$. [(c) and (d)] The four-times magnification of the squared region in (a) and (b). [(e) and (f)] The four-times magnification of the squared region in (c) and (d). Run D.

Figure 8 is a side-by-side comparison between the spatial distributions of zero-acceleration points and inertial particles for $S_{\eta}=1.9$ in Run D. It is observed that these two distributions are spatially well correlated at all length scales, and this correlation persists unaltered as time advances. The multiscale nature of holes of inertial particles and that of zero-acceleration points look surprisingly similar.

For a quantification, we calculate the PDF of hole areas of zero-acceleration points by the same method as we did for inertial particles, and plot it (thick solid curve) in Fig. 7. In both cases of $\mathcal{L} / \eta$, the PDF of hole areas of zeroacceleration points is in excellent agreement with the PDF of hole areas of inertial particles when $S_{\eta}$ is such that the range of scales between $\ell_{\min }$ [see (9)] and $\ell_{\max }$ [see (10)] is fully within the inertial range.

In Ref. 7, we attempted an explanation of turbulent clustering of inertial particles without reference to coarse-grained vorticity but solely in terms of zero-acceleration points and the fact that, like vorticity in two dimensions, they are predominantly (in a statistical and asymptotic sense defined in Ref. 7) swept by the large-scale velocity field. Instead, in this article, we have presented an explanation for particle clustering that depends crucially on the fact that coarse-grained vorticity is swept by the fluid velocity. One advantage of the explanation based on zero-acceleration points is that their sweeping by large-scale eddies is valid in three-dimensional flows as well as two-dimensional turbulence, whereas vorticity in three-dimensional flows is not only swept but also stretched. The second advantage of the acceleration approach is that it may be more appropriate than the coarse-grained vorticity approach to explain the presence or absence of particle clustering in various inhomogeneous turbulent flows.

\section{Physical explanation of the coincidence of two distributions}

The argument developed in Ref. 7 to explain the coincidence between distributions of particles and zeroacceleration points is summarized as follows. The velocity $\boldsymbol{v}_{p}$ of an inertial particle with $\tau_{p}$ sufficiently smaller than $\tau_{\eta}$ is well approximated by $\boldsymbol{u}-\tau_{p} \boldsymbol{a},{ }^{16}$ where $\boldsymbol{u}$ and $\boldsymbol{a}$ are the fluid velocity and acceleration at the particle position $\boldsymbol{x}_{p}$. Therefore, at a point where $\boldsymbol{a}=0$, inertial particles tend to move with the fluid velocity. On the other hand, as demonstrated in Ref. 7, the acceleration field, and in particular its zeroacceleration points, move on average with same fluid velocity $\boldsymbol{u}$. Consequently, an inertial particle and a coincident zero-acceleration point will move together for a while, whereas a particle at a point where $\boldsymbol{a}$ is nonzero will swiftly move toward other values of $\boldsymbol{a}$ because $\boldsymbol{v}_{p}=\boldsymbol{u}-\tau_{p} \boldsymbol{a}$. Note that this argument does not imply that zero-acceleration points attract inertial particles. Zero-acceleration points are simply more sticky for particles than other regions where the acceleration takes larger values. Zero-acceleration points being clustered, the fact that particles move from one zeroacceleration point to another and spend some significant time with them implies that zero-acceleration point clusters trap inertial particles. As a result, particles cluster in a way that reflects the clustering of zero-acceleration points. It is important to stress that both clusters of zero-acceleration points and of inertial particles are not static.

The above reasoning is limited to $\tau_{p}$ much smaller than $\tau_{\eta}$. However, the coincidence of the two clusters is observed even when $\tau_{p}$ is comparable to or much larger than $\tau_{\eta}$. This superficial inconsistency is resolved as follows. In Refs. 12 and 18 , we introduced the idea that velocity stagnation points have a length scale and a time scale tagged to them and that these scales can take many different values within the powerlaw energy spectrum's range. Following this idea, it might be natural to expect zero-acceleration points also to have a time scale $\tau_{a}$ tagged to them. This time scale might be defined as

$$
\tau_{a} \equiv\left(\left|\lambda_{1}\right|^{2}+\left|\lambda_{2}\right|^{2}\right)^{-1 / 4}
$$

where $\lambda_{1}$ and $\lambda_{2}$ are the eigenvalues of the matrix $\partial a_{i} / \partial x_{j}$ because these gradients give an indication of the extent of the region around $\boldsymbol{a}=0$ where $\boldsymbol{a}$ remains close to 0 . We observe in Figs. 9(a), 9(d), and 9(g) that the subcluster of zeroacceleration points that have values of $\tau_{a}$ larger than a certain threshold resembles the entire cluster of zero-acceleration points for any threshold not much larger than $\mathcal{T}$ over a range of coarse-graining spatial resolutions that is a subrange of the inertial range. We also observe in Fig. 9 that the subcluster of zero-acceleration points, plotted in Figs. 9(b), 9(e), and 9(h), 


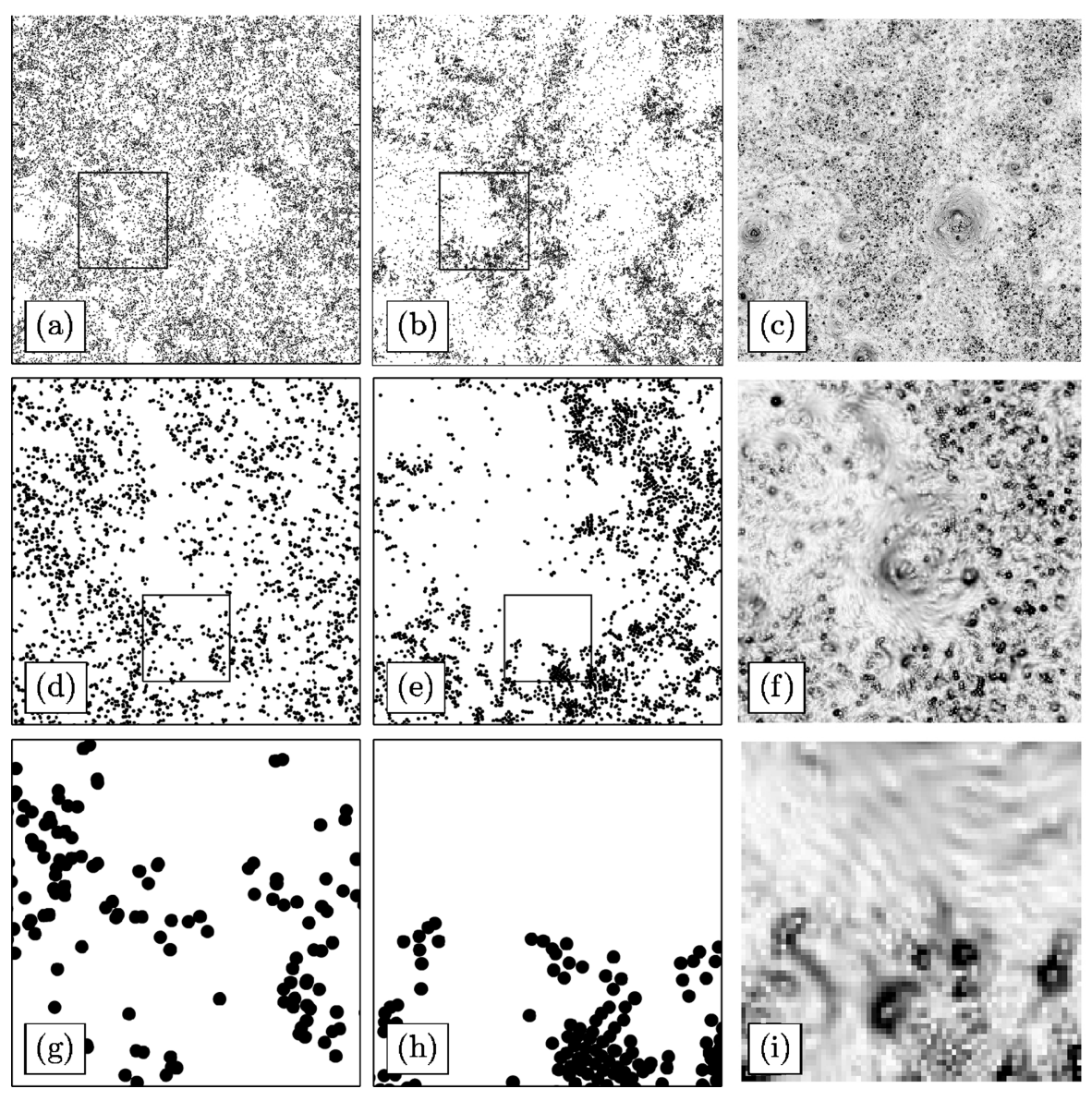

Phys. Fluids 18, 115103 (2006)

FIG. 9. [(a), (d), and (g)] Zeroacceleration points with time scales $\tau_{a}$ larger than $12.8 \tau_{\eta}$. [(b), (e), and (h)] Zero-acceleration points with time scales $\tau_{a}$ smaller than $12.8 \tau_{\eta}$. [(c), (f), and (i)] Acceleration magnitudes are plotted in shades of gray, darker shades corresponding to higher magnitudes. Plotted times and regions are the same as in Fig. 8: full side lengths of the plots are $7 \mathcal{L} \approx 200 \eta$ in (a)-(c), $3.5 \mathcal{L} \approx 100 \eta$ in (d) $-(\mathrm{f})$, and $1.8 \mathcal{L}$ $\approx 50 \eta$ in $(\mathrm{g})-(\mathrm{i})$. Run D. with $\tau_{a}$ smaller than the threshold, is located only in high acceleration regions, i.e., darker regions in Figs. 9(c), 9(f), and 9(i). As a result, we may expect inertial particles of relaxation time $\tau_{p}$ to be trapped by subclusters of zeroacceleration points with $\tau_{a}$ much larger than $\tau_{p}$ because $|\boldsymbol{a}|$ takes smaller values around such zero-acceleration points than around zero-acceleration points with $\tau_{a}<\tau_{p}$. Hence, zero-acceleration points with $\tau_{a} \gg \tau_{p}$ are stickier for particles of relaxation time $\tau_{p}$ than zero-acceleration points with $\tau_{a}$ $<\tau_{p}$. This explains why inertial particles of any relaxation time $\tau_{p}$ not much larger than $\mathcal{T}$ cluster in a way that reflects the clustering of zero-acceleration points over a broad range of coarse-graining resolutions. Note that when $\tau_{p} \gtrsim \mathcal{T}$, particles do not cluster in a way that reflects zero-acceleration point clustering because there is no zero-acceleration points with $\tau_{a} \gg \mathcal{T}$.

This explanation raises two questions: (i) Is our explanation consistent with the disappearance of inertial particle clustering when $\tau_{p} / \tau_{\eta} \rightarrow 0$ ? (ii) Why are large holes in the particles' clustering absent when $\tau_{p}$ is much smaller than $\mathcal{T}$ but larger than $\tau_{\eta}$ ? Concerning the first question, indeed, when $\tau_{p}=0$, there is no clustering at all and $\boldsymbol{v}_{p}=\boldsymbol{u}$. The difference between $\boldsymbol{v}_{p}$ and $\boldsymbol{u}$ when $\tau_{p}$ is nonzero yet much smaller than $\tau_{\eta}$ is approximately $-\tau_{p} \boldsymbol{a}$. Hence, $-\tau_{p} \boldsymbol{a}$ limits the "stickiness" of zero-acceleration points and regions surrounding them. When $\tau_{p}=0$, the entire space is "sticky" because $-\tau_{p} \boldsymbol{a}=0$ and particles are fluid elements. When $\tau_{p} \neq 0$, a "sticky" region surrounding a zero-acceleration point may be defined as the region where $\tau_{p}|\boldsymbol{a}|$ is much smaller than a typical velocity of the fluid, e.g., $u^{\prime}$. The size $\ell_{a}$ of this region is a function of both $\tau_{a}$ and $\tau_{p}$, in fact an increasing function of $\tau_{a}$ (because increasing values of $\tau_{a}$ reflect shallower acceleration gradients at $\boldsymbol{a}=0$ ) and a decreasing function of $\tau_{p}$. As $\tau_{p}$ decreases far below $\tau_{\eta}$, the clustering disappears because $\ell_{a}$ becomes increasingly large. Similarly, concerning the second question, as $\tau_{p}$ increases above $\tau_{\eta}, \ell_{a}$ gradually decreases, and as a consequence, the size of the relatively empty holes increases. Incidentally, in such cases in which $\tau_{p}$ is slightly larger than $\tau_{\eta}$, particles' holes exist only in regions where $|\boldsymbol{a}|$ takes extremely large values, i.e., extremely "slippery" regions for particles.

\section{CONCLUDING REMARKS}

All the past works (e.g., Refs. 3-5 among others) showing that particles move away from vortical regions and spend much time in straining regions were carried out numerically or experimentally in turbulent flows of limited Reynolds number and therefore with an insufficiently defined range of self-similar scales. In contrast, our turbulence has a $k^{-5 / 3}$ energy spectrum defined over nearly two decades and we show that the clustering in such turbulence does indeed require consideration of the entire range of scales to be explained. Furthermore, what these previous studies did not make clear is the central importance of the sweeping in the way that high Reynolds number turbulence clusters inertial particles. In fact, if there were no sweeping, the clustering of inertial particles would not occur as it does. Indeed, as demonstrated 
in Ref. 7, the clustering of inertial particles is qualitatively different in kinematic simulations of two-dimensional turbulence with the $k^{-5 / 3}$ energy spectrum where there is no sweeping but where there are well-defined high enstrophy and high-strain rate regions as in our two-dimensional DNS turbulence. And in both simulations, high strain rate regions do not explain clustering because there are many cases of clustered inertial particles (depending on Stokes and Reynolds numbers) where the ratio of the enstrophy averaged over all inertial particles to the square of the strain rate also averaged over all inertial particles is equal to 2 , the value taken by this ratio when these averages are taken over all space of a statistically homogeneous turbulence (see Ref. 7). One would have expected this ratio to be much smaller than 2 if the particle clustering could be simply explained solely in terms of regions of high strain rate.

Of equally central importance to the clustering of inertial particles, as we show here, is the cluster of zero-acceleration points. The effect of zero-acceleration clusters was missed in previous studies because the knowledge of the importance of the concepts of stagnation points (whether zero-acceleration or zero-velocity) and of their specific properties (as opposed to the properties of the enstrophy and strain rate fields) was missing (see Refs. 7, 12, and 18 and references therein for more details on the relatively recent development of this new approach). If there were no acceleration clusters, the clustering of inertial particles would not occur as it does in this two-dimensional turbulence. As a result of sweeping of the acceleration field by the velocity field, inertial particles cluster in a way that closely mimics the zero-acceleration points cluster. Important in this thinking is our concept of stickiness of zero-acceleration points. The stickiness stems from the fact that zero-acceleration points move, together with nearby inertial particles, in the local velocity induced by larger eddies.

We now summarize our conclusions. A first important result of this study is that particle clustering occurs at all Stokes numbers,

$$
\alpha<S_{\eta}<\beta\left(\mathcal{T} / \tau_{\eta}\right)
$$

where $\alpha$ and $\beta$ are given in (11) for the considered turbulence.

Second, because the particle voids cannot be explained in terms of bare vorticity and strain rate concepts, we have explained them in terms of coarse-grained vorticity and scale-dependent Stokes numbers $S(\ell)=\tau_{p} / T(\ell)$, where $T(\ell)$ varies between $\tau_{\eta}$ and $\mathcal{T}$ according to length scale $\ell$. A central concept of this explanation is the existence of resonant eddies over a decade-long range of length scales bounded from below and above by $\ell_{\min }$ and $\ell_{\max }\left(\ell_{\max } / \ell_{\min } \approx 30\right)$. These are eddies that have time scales $T(\ell)$ such that $S(\ell)$ is neither too large nor too small. The statistical self-similarity of eddies in turbulence with a $k^{-5 / 3}$ energy spectrum implies a statistical self-similarity of clusters of inertial particles that is reflected in the power-law shape of the PDF $P(A)$ of void areas $A$. Specifically, $P(A) \sim A^{-5 / 3}$ over a range $A_{\min }<A$ $<A_{\max }$ with $A_{\max } / A_{\min }=\left(\ell_{\max } / \ell_{\min }\right)^{2} \approx 1000$ for all Reynolds numbers $\mathcal{L} / \eta$ larger than $\ell_{\max } / \ell_{\min }$. We have proposed a simple model based on resonant eddies that implies that this exponent $-5 / 3$ follows from $E(k) \sim k^{-5 / 3}$.

The third contribution of our study is the bridging of the gap between previous explanations of inertial particle clustering that are based on vorticity, and our recent claim ${ }^{7}$ that inertial particles cluster, irrespective of the Stokes number [in the range (21)], in a way that directly reflects the clustering of zero-acceleration points. This gap is bridged in the sense that multiscale eddies can explain the appearance of particle voids, whereas acceleration clusters can directly explain particle clusters. Finally, we have developed and clarified the explanation of Ref. 7 for the clustering of inertial particles over many length-scale resolutions solely in terms of zero-acceleration point clusters, and we have shown how this explanation is valid over the entire Stokes number range (21). This conclusion, that properties of inertial particle clustering can be explained solely in terms of fluid acceleration, may have broad applications to particle clustering in various turbulent fields, where the centrifugal effect of eddies is not the only source of fluid acceleration.

\section{ACKNOWLEDGMENTS}

This research was partially supported by the Japanese Ministry of Education, Science, Sports and Culture, Grantin-Aid for Young Scientists, and by the 21st Century Program in Kyoto University on Complex Functional Mechanical Systems.

${ }^{1}$ P. A. Vaillancourt and M. K. Yau, "Review of particle-turbulence interactions and consequences for cloud physics," Bull. Am. Meteorol. Soc. 81, 285 (2000).

${ }^{2}$ J. K. Eaton and J. R. Fessler, "Preferential concentration of particles by turbulence,” Int. J. Multiphase Flow 20, 169 (1994).

${ }^{3}$ K. D. Squires and J. K. Eaton, "Preferential concentration of particles by turbulence," Phys. Fluids A 3, 1169 (1991).

${ }^{4}$ L. P. Wang and M. R. Maxey, "Settling velocity and concentration distribution of heavy-particles in homogeneous isotropic turbulence," J. Fluid Mech. 256, 27 (1993).

${ }^{5}$ T. S. Yang and S. S. Shy, "Two-way interaction between solid particles and homogeneous air turbulence: Particle settling rate and turbulence modification measurements," J. Fluid Mech. 526, 171 (2005).

${ }^{6}$ G. Boffetta, F. De Lillo, and A. Gamba, "Large scale inhomogeneity of inertial particles in turbulent flow," Phys. Fluids 16, L20 (2004).

${ }^{7}$ L. Chen, S. Goto, and J. C. Vassilicos, "Turbulent clustering of stagnation points and inertial particles," J. Fluid Mech. 553, 143 (2006).

${ }^{8}$ M. Maxey and J. Riley, "Equation of motion of a small rigid sphere in a nonuniform flow," Phys. Fluids 26, 883 (1983).

${ }^{9}$ R. H. Kraichnan, "Inertial ranges in two-dimensional turbulence," Phys. Fluids 10, 1417 (1967).

${ }^{10}$ C. E. Leith, "Diffusion approximation for two-dimensional turbulence," Phys. Fluids 11, 671 (1968).

${ }^{11}$ G. K. Batchelor, "Computation of the energy spectrum in two-dimensional turbulence," Phys. Fluids 12, 233 (1969).

${ }^{12}$ S. Goto and J. C. Vassilicos, "Particle pair diffusion and persistent streamline topology in two-dimensional turbulence," New J. Phys. 6, 65 (2004).

${ }^{13}$ A. B. Kostinski and R. A. Shaw, "Scale-dependent droplet clustering in turbulent clouds," J. Fluid Mech. 434, 389 (2001).

${ }^{14}$ L. Landau and E. Lifshitz, Statistical Physics (Pergamon, New York, 1980).

${ }^{15} \mathrm{C}$. Tchen, "Mean values and correlation problems connected with the 
motion of small particles suspended in turbulent fluid," Ph.D. dissertation, University of Delft, Holland (1947).

${ }^{16}$ G. Falkovich and A. Pumir, "Intermittent distribution of heavy particles in a turbulent flow," Phys. Fluids 16, L47 (2004).
${ }^{17}$ S. Goto and S. Kida, "Reynolds-number dependence of line and surface stretching in turbulence: Folding effects," J. Fluid Mech. (submitted).

${ }^{18}$ L. Rossi, J. C. Vassilicos, and Y. Hardalupas, "Multiscale laminar flows with turbulentlike properties," Phys. Rev. Lett. 97, 144501 (2006). 